Adrián Schopf y Juan Carlos Marín (editores), Lo público y lo privado en el derecho. Estudios en homenaje al profesor Enrique Barros Bourie (Santiago: Thomson Reuters, 2017).

PRESENTACIÓN

\title{
UN JURISTA SINGULAR
}

El miércoles 9 de agosto de 2017, en el Aula Magna de la Facultad de Derecho de la Universidad de Chile, se llevó a cabo la presentación del libro Lo público y lo privado en el derecho. Estudios en homenaje al profesor Enrique Barros Bourie. Con ello, mediante un acto académico, la ciencia jurídica nacional y la práctica profesional rindieron un homenaje a uno de los más destacados juristas chilenos. La presentación de la obra estuvo a cargo de los profesores María Agnes Salah Abusleme, José Miguel Huerta Molina y Hernán Corral Talciani. En la última parte de la ceremonia tomó la palabra el homenajeado, el profesor Enrique Barros. Reproducimos a continuación estas intervenciones.

María Agnes Salah Abusleme

"Un profundo amor por el derecho" 168

José Miguel Huerta Molina

"Las facetas de un jurista".

Hernán Corral Talciani

"Una personalidad singular"

Enrique Barros Bourie

"Un profundo agradecimiento" 


\section{UN PROFUNDO AMOR POR EL DERECHO}

\section{María Agnes Salah Abusleme}

M uy probablemente se podría reflexionar en estos momentos acerca del aporte del profesor Barros a la teoría del derecho o al derecho privado chileno. Yo simplemente daré mi testimonio como estudiante del profesor Enrique Barros y académica de la facultad. ${ }^{1} \mathrm{Ha}-$ blaré desde los afectos. Espero no defraudar a los organizadores de esta ceremonia con el giro que daré al programa.

No es casualidad que seis compañeros de mi curso de derecho civil con el profesor Barros nos desempeñemos en la actualidad como profesores del ramo. No es casualidad que, a pesar de tener fuertes inclinaciones por otras áreas del derecho, sea derecho civil el ramo que decidimos enseñar. No es casualidad que muchos otros de los profesores del Departamento de Derecho Privado hayan sido estudiantes de los famosos ciclos de Barros o hayan sido formados por discípulos del mismo. Finalmente, tampoco es casualidad que muchos de los profesores de derecho civil, hubieran o no estudiado en esta facultad, miren al profesor Barros con una conmovedora admiración.

Cuando algunas personas me han preguntado, debo decir que con cierta sospecha, acerca de la desmedida inclinación de mi generación hacia el derecho civil, no he dudado un segundo en mirar al profesor Barros como la respuesta. No puedo negar que tuve extraordinarios profesores durante mis años de estudio en esta facultad, pero quienes tuvimos a Barros de profesor sabemos que su presencia marcó un punto de inflexión en nuestras vidas académicas.

Vale la pena preguntarse por qué.

María Agnes Salah Abusleme. Abogada. LLM en Harvard University. Doctora en derecho por la Universidad de Chile. Profesora de la Facultad de Derecho de la Universidad de Chile. Email: msalah@derecho.uchile.cl. La autora quiere señalar: "Cuando recibí el desafiante encargo de pronunciar algunas palabras en esta ceremonia pude compartir ideas, impresiones y anécdotas con los profesores Fernando Atria, Cristián Banfi, Nicolás Rojas y Francisca Román, lo que ciertamente benefició el resultado alcanzado".

${ }^{1}$ Facultad de Derecho de la Universidad de Chile (n. del e.). Las siguientes referencias a "la facultad" refieren al mismo espacio. 
Primero, nos marcó su profundo amor por el derecho, una carrera a la que muchos de nosotros entramos con dudas; una carrera que parecía mucho menos loable que la labor de los médicos al salvar vidas; una carrera que quedaba pequeña frente a la monumental obra de un artista. Sin embargo, Barros nos mostraba la dignidad de esta carrera en cada una de sus clases. Como por arte de magia, el derecho ya no parecía un objeto de estudio infame, era una materia que podía ser profundamente humana, y en cierta forma — mucho más sutil para el caso del derecho civil— también podía contribuir a salvar vidas. Esto es lo que nos mostró el profesor Barros día a día, en cada una de las clases de esos cuatro años: que el derecho podía ser una gran herramienta para hacer de este mundo algo mejor.

Seguidamente, nos enseñó el sentido de la excelencia. Este abstracto concepto se hacía, clase a clase, concreto y con contenido. Cada una de sus lecciones era una muestra indubitable de que las cosas se podían hacer en serio; de que la academia se podía tomar en serio; y de que se podía marcar una diferencia en la forma de enseñar en la universidad. El profesor Barros nos demostró con su ejemplo que la universidad de excelencia existía, que era un ideal que podía y debía alcanzarse.

Como si lo anterior no fuera suficiente, nos enseñó la necesidad de comprender al derecho civil en un contexto mayor, el de la vida en sociedad. Más de algún dolor de cabeza nos causaron sus seminarios, en que se profundizaban — como si hubiéramos tenido un punto desde el cual comenzar - las enseñanzas de Aristóteles, Santo Tomás, Kant, Gehlen, Malinowski y Pashukanis. Sin entender mucho, nos sumergíamos en la vida de los nuer, sin que pudiéramos explicarle al mundo de qué forma esta milenaria tribu africana se conectaba con el Código Civil chileno. Varios años me llevaron para entender el sentido de todas estas lecturas y la forma en que eran trascendentales para el derecho.

El origen de esta aproximación no era una casualidad. El profesor Barros, antes de dedicarse al derecho civil, tuvo una larga trayectoria en el área de teoría del derecho. Sin embargo, él vio con algo de espanto que "el antiguo dinamismo de los juristas privatistas" había sido reemplazado por una doctrina que "sin convicción" repetía antiguas fórmulas dogmáticas. ${ }^{2}$ Así, tomó como lema de batalla restablecer la dignidad a esta importante área del derecho, mejorar de esta sutil forma al país. Y lo logró.

${ }^{2}$ Enrique Barros, "Método científico y principios jurídicos del gobierno constitucional", Estudios Públicos 2 (1981): 8. 
Con mucha generosidad nos transmitió todo lo que sabía, pero también lo que intuía. Sólo con el pasar del tiempo nos pudimos dar cuenta de que muchas de las cosas aprendidas en sus clases en realidad eran teorías en elaboración del profesor Barros. Pero la fuerza de sus argumentos nunca nos dio luces de lo que estaba realmente ocurriendo en la sala de clases. Con la verdadera humildad que sólo los maestros tienen con sus estudiantes, nos consideraba como interlocutores relevantes en la confirmación o refutación de sus incipientes hipótesis.

Con la misma generosidad formó a decenas de ayudantes, todos diferentes en muchos sentidos; escribió elogiosas cartas de recomendación en cada uno de nuestros emprendimientos y se alegró con todos nuestros éxitos y logros, académicos y personales. Con la misma nobleza asiste cada año a las Jornadas Nacionales de Derecho Civil, realizando respetuosas y constructivas críticas para que nuestra disciplina progrese más. Así nos mostró que la academia de tiempo completo se podía y se debía hacer.

La relación entre un maestro y un estudiante es una relación destinada a transformarse. Si es exitosa, el estudiante se emancipará y será un igual. En nuestra a veces triste realidad, este tránsito no es sencillo y deviene, ya sea en una relación de vasallaje o en una ruptura irreparable. En el caso del profesor Enrique Barros, fue posible completar exitosamente este tránsito.

Ello no es sino un reflejo de su profunda convicción de que lo relevante no es pensar igual, sino que pensar bien. Esto es lo que constituye una verdadera comunidad académica, aquel espacio en que el disenso respetuoso beneficia a cada uno de sus integrantes.

Para que no piensen que este discurso carece de sentido crítico, podemos decir que algunos defectos tiene el profesor Barros. Imposible olvidar los fuertes golpes en nuestras cabezas o espaldas - como metáforas de un cercano saludo- , las posturas casi circenses en el podio de clases mientras tratábamos de asimilar el contenido de la buena fe, las indiscretas carcajadas en los más inesperados momentos y los códigos sumergiéndose en charcos de agua derramada en la sala de clases. Nunca le perdonaremos haber roto el regalo entregado en la comida de fin de ciclo en el momento exacto de su apertura.

Pero siempre le agradeceremos por habernos formado con tanto cariño y dedicación. Su obra perdurará, sus libros perdurarán. Pero, más importante aún, perdurarán los cimientos asentados en el Departamento de Derecho Privado, lo que, a mi juicio, constituye un acervo inmaterial de valor mucho mayor que el de cualquier tratado. 


\section{LAS FACETAS DE UN JURISTA}

\section{José Miguel Huerta Molina}

M e siento honrado de ser uno de los que hablan en este homenaje. Estoy honrado porque don Enrique es, a mi juicio, el académico del derecho más relevante de nuestro tiempo y uno de los más influyentes de la historia de Chile. Estoy especialmente honrado porque don Enrique es el profesor que más ha marcado mi vida profesional.

Conocí a don Enrique hace más de 22 años, en marzo de 1995. En mi primera clase de mi primer día de clases en nuestra facultad. Recuerdo muy claramente la impresión que me causó esa primera clase de derecho civil. Como estudiante de región, recién salido del colegio, tenía altas expectativas de lo que podía ser un profesor de derecho de la Universidad de Chile. Pero lo que encontré superó con creces lo que esperaba y me hizo pensar que había sido un acierto estudiar derecho.

Durante los cuatro años que duró el ciclo de derecho civil esa impresión inicial siempre fue confirmada. Qué lujo era llegar a nuestra escuela los lunes, martes y jueves, a las 8:30, a escuchar y participar en la cátedra de don Enrique. El contenido, la claridad, lo entretenido y la inteligencia de cada una de las cientos de clases que nos hizo significaron que fuera elegido el mejor profesor de nuestro ciclo. Significó también que fuera, para mí, el mejor profesor que he tenido, tanto en Chile como en el extranjero.

Pero esta brillantez de su cátedra no es sólo fruto del talento. Estoy cierto de que detrás de cada una de sus clases, además de un profundo amor por la enseñanza y el derecho, hay largas horas de preparación. Hay mucha dedicación. Hay un enorme esfuerzo.

Pero la influencia de Enrique Barros va mucho más allá de ser un brillante académico. Su labor como formador de profesores es portentosa, creando una verdadera escuela de académicos que han crecido bajo su influencia. Durante los cuatro años de nuestro ciclo, tres ayudantes de don Enrique nos hicieron algunas clases. Ellos fueron Andrés Jana,

José Miguel Huerta Molina. Abogado. Máster en derecho por la Universidad de Stanford. Profesor de la Facultad de Derecho de la Universidad de Chile. Email: jmhuerta@claro.cl. 
Juan Carlos Marín y Francisco González, todos hoy destacados profesores de derecho.

Pero no sólo eso.

Como muchos de ustedes saben, Enrique Barros instauró la tradición de realizar talleres complementarios a sus clases, a cargo de ayudantes. Dichos talleres han sido también cuna de destacados académicos de nuestro país. Sin ir más lejos, tuve la fortuna de que mi primera ayudante de talleres fuera Daniela Accatino, y luego que me hicieran esos talleres los actuales profesores Mauricio Tapia y José Miguel Valdivia. Este semillero de ayudantes que luego son profesores, y que a su vez forman futuros profesores, ha tenido una expansión geométrica. No me atrevo a nombrarlos a todos, porque de seguro se me van a quedar muchos fuera, pero en esta sala veo a varios de ellos, como mi amiga María Agnes Salah, y mis amigos Paulo Montt, Rodrigo Gil, Nicolás Rojas, Adrián Schopf, Felipe de Marinis y varios otros que fueron alumnos de don Enrique o alumnos de sus ex alumnos.

Pero la influencia formativa de Enrique Barros trasciende el mundo académico.

Son muchos los jueces, funcionarios públicos, políticos o abogados practicantes que hoy tienen un rol relevante en nuestro país y que fueron formados por él o por miembros de su escuela. Varios de ellos están hoy presentes.

Pero Enrique Barros no sólo es uno de los más destacados académicos de nuestro tiempo y un gran formador de profesionales. Don Enrique ha tenido un rol muy relevante en la judicatura de Chile como integrante de la excelentísima Corte Suprema y como árbitro. Ha tenido también una participación importante en el desarrollo de políticas públicas de nuestro país; por ejemplo, mediante su participación en el Centro de Estudios Públicos, en el Colegio de Abogados y otras instituciones que ha presidido o ha integrado.

Pero no sólo eso.

Don Enrique está lejos de ser un mero teórico del derecho o de las políticas públicas. Pese a que me ha dicho que lo que más lo apasiona desde temprana edad es la academia, ha sido y sigue siendo uno de los mejores abogados practicantes de Chile, no sólo en la asesoría preventiva de clientes en las más diversas áreas, sino también en terreno, como abogado litigante. He tenido la oportunidad de trabajar con él y con su 
equipo en diversos casos de la mayor complejidad, ya sea pidiéndole informes en derecho, actuando como copartes, como contraparte, o sencillamente recurriendo a su buen consejo. Cuando se trata de enfrentar un conflicto nuevamente he encontrado en don Enrique a un profesional que trabaja con pasión e investiga y analiza hasta la última arista del problema. No sólo eso, he encontrado a un abogado con el mayor sentido práctico y estratégico, y a un buen conocedor de la lógica de nuestra judicatura.

Pese a este talento y a ser uno de los abogados practicantes más prestigiosos de Chile, don Enrique siempre se ha dedicado con pasión a la cosa pública, y en especial a la universidad. Durante mucho tiempo me pregunté por qué alguien de su talla estaba dispuesto a invertir gran parte de su vida en enseñar a un grupo desconocido de jóvenes. Creo que con el tiempo lo entendí.

En la última clase de nuestro ciclo, don Enrique nos dijo que debíamos gratitud a nuestra facultad y que teníamos un deber de entregar a otros lo que habíamos recibido en estas aulas. Don Enrique ya nos había dado esta lección durante cuatro años, a través de su ejemplo. Ese sentido de gratitud y de que existe un deber de retribuir lo que uno recibió de sus maestros es la mayor enseñanza de Enrique Barros, y es, creo, la razón de la enorme influencia que ha ejercido en nuestro país. Ese sentido de gratitud es el que ha llevado a que varias decenas de quienes fueron sus alumnos, o alumnos de sus alumnos, o alumnos de los alumnos de sus alumnos sean hoy destacados servidores en los más diversos ámbitos.

En tiempos en que en Chile muchos parecen creer que los problemas se solucionan desde arriba, que los países avanzan sólo por el impulso del Estado, surgen figuras como la de Enrique Barros, que nos enseñan que los individuos son más importante que los sistemas, que el ejemplo, el cariño y la dedicación de una sola persona pueden generar un círculo virtuoso de cientos de otras dispuestas a esforzarse día a día en hacer las cosas bien y en ayudar y enseñar a otros.

Igual que muchos de los que están hoy en esta sala, he tenido el privilegio de conocer a don Enrique, de ser su alumno y recibir su influencia. Estoy consciente de que este privilegio conlleva un deber. Espero estar a la altura del mismo. 


\section{UNA PERSONALIDAD SINGULAR}

\section{Hernán Corral Talciani}

L

os editores del libro colectivo me han solicitado que diga algunas

palabras sobre el homenajeado en mi calidad de académico y docente en materias propias del derecho civil.

Me parece que puedo intentar describir la singular personalidad y la fecunda labor de Enrique Barros, tomando ocasión justamente de algunas características del volumen que presentamos en su homenaje.

Diría que el libro es reflexivo, dialogante, innovador y tradicional a la vez, multifacético y de una estética elegante pero también cálida y acogedora. Barros Bourie encarna, a mi juicio, todas estas cualidades en su persona y en su trabajo académico.

Sin duda, Enrique es un hombre reflexivo, que confía en la racionalidad humana para obtener, mediante argumentos coherentes y sensatos, soluciones justas a los problemas jurídicos que se plantean en la sociedad. Es también un hombre de fe, pero muy consciente de que una visión trascendental del sentido de lo humano no coarta ni limita, sino, más bien, da nuevos bríos a la inteligencia.

Esa misma reflexividad - y la profundidad de su cultura y de sus conocimientos jurídicos, que es por todos sus colegas reconocida- no le impide ser un académico que se plantea, antes que en confrontación o beligerancia, en actitud de diálogo, de saber escuchar al otro para lograr una síntesis o al menos una delimitación de los puntos de controversia. De hecho, Enrique suele decir que, para encontrar las respuestas a los problemas, lo primero es formular correctamente las preguntas.

La contribución del profesor Barros a la dogmática civil ha sido innovadora, pero de aquella innovación que no desprecia la doctrina tradicional. Su obra hasta ahora señera, el Tratado de responsabilidad extracontractual, que mereció un encendido elogio de Fernando Pantaleón, el conocido jurista español, como el mejor tratado escrito en lengua castellana, contiene un tratamiento avanzado de esta importante temática, pero, como podrá comprobar cualquier lector, no se ha des-

Hernán Corral Talciani. Abogado. Doctor en derecho por la Universidad de Navarra. Profesor de derecho civil, Universidad de los Andes. Email: hcorral@ uandes.cl. 
deñado lo clásico: se cita profusamente la obra de Alessandri, así como otras contribuciones tanto del derecho comparado como nacional. El profesor Barros tampoco gusta de la novedad por la novedad, y muchas veces prefiere atenerse a las construcciones dogmáticas que ya se han consolidado, si éstas funcionan razonablemente bien en la práctica.

Pero no sólo con su prolífica obra publicada ha contribuido Enrique Barros al desarrollo de la civilística nacional. Le debemos mucho más, porque ha sido un constante impulsor de iniciativas académicas que han fortalecido el cultivo y el progreso de esta área del saber jurídico.

Pienso que a él le debemos gran parte, si no todo, del florecimiento que hoy vive nuestra disciplina, con muchos jóvenes talentosos que han realizado doctorados en diferentes universidades, y que de una u otra manera están vinculados al magisterio del profesor Barros, pues, o han sido sus alumnos o reconocen que han recibido el influjo benefactor de su magisterio. Personalmente $-\mathrm{y}$ advierto que no pretendo incluirme en las nuevas generaciones de civilistas, porque de esto ya han pasado más de 25 años-, recuerdo con gratitud que, al regresar de mi doctorado el año 1990, el profesor tuvo la gentileza de recibirme en su oficina de director del Departamento de Derecho Privado de esta facultad. A pesar de que no me conocía, tuvo palabras y gestos de estímulo que en ese momento fueron decisivos para encauzar mi vocación académica.

Hay que pensar que en aquellos años lo que "la llevaba" (en la jerga juvenil actual) en materia de renovación y nuevos aires, era el derecho público, que aparecía como la gran estrella en cuanto defensor del "administrado" frente a los abusos del poder estatal, así como la exportación de teorías como la del efecto horizontal de los derechos y la aplicación directa de la Constitución. Gracias al llamado "recurso" de protección parecía que ya el Código Civil no tenía mayor importancia, y que todo podía resolverse mediante la invocación de una o más garantías constitucionales. Juicios de arriendo, acciones reivindicatorias, pago de lo no debido, todo se reconducía a la Constitución según el parecer de los magistrados que integraran las salas de las cortes. Enrique Barros fue uno de los primeros que advirtieron que convertir esa acción de cautela urgente, diseñada para evitar la autotutela, en la panacea para todos los problemas jurídicos, incluidos los propios del derecho privado, era un mal camino que empobrecía la dogmática del derecho y conducía a una inevitable vulgarización jurídica. 
Por diferentes vías, incluida la dirección de una tesis de licenciatura de los hoy prestigiosos abogados y profesores Juan Carlos Marín y Andrés Jana, sobre recurso de protección y contratos, además de su servicio como abogado integrante de la Corte Suprema por seis años continuos, Enrique ha sido uno de los factores fundamentales para que hoy podamos decir que aquella fiebre por resolver los problemas de derecho privado vía recurso de protección está en franca retirada. Así, se han vuelto a apreciar los criterios propios de una dogmática civil, que a la vez se ha ido enriqueciendo con los escritos de más y más profesores dedicados a su cultivo.

Ese esfuerzo por estimular el estudio colectivo del derecho civil es también algo que debemos al carácter innovador del profesor Barros. Son un hito histórico, que incluso entran en el terreno de lo legendario, aquellas Primeras Jornadas de Derecho Civil que él convocó en las Termas de Jahuel el año 1989, y cuyos resultados se convirtieron en dos libros que fueron editados bajo su dirección por la Editorial Jurídica de Chile. Cuando en 2005, por invitación del entonces decano de la Facultad de Derecho de la Universidad Austral de Valdivia, Juan Andrés Varas, otro de sus aventajados discípulos, se habló de proseguir la labor de esas jornadas conformando un consorcio de facultades de derecho que pudiera asegurarles continuidad, Enrique Barros se sumó entusiastamente a la iniciativa e incluso aceptó dar la conferencia inaugural. Me consta que desde entonces, con una activa participación en el comité del consorcio sostenedor de las jornadas, y como ponente y activo participante, siempre tomando notas en un enigmático cuaderno, las ha acompañado y apoyado por más de una década. Esas jornadas han dado lugar a la obra Estudios de derecho civil, que ya va en su duodécimo volumen, el que se presentará, Dios mediante, en las próximas XV jornadas, como ha sido la regla en todo este tiempo.

Entre sus muchas contribuciones a nuestra dogmática civil, podemos destacar la relevancia que el profesor Barros le ha dado a la justicia correctiva en la conformación de la lógica y finalidad propia del derecho privado, y que le confiere una autonomía frente a los intentos de instrumentalización propiciados por un utilitarismo de corte kelseniano o, más recientemente, por la escuela del Laws and Economics. Esta misma justicia correctiva es el concepto basilar, si bien no único, sobre el que levanta toda su teoría de la responsabilidad extracontractual expuesta en su magnífico tratado. 
En el último tiempo, cabe destacar sus estudios y análisis sobre el derecho de los contratos en línea con la tendencia propiciada por la Convención de Viena, inspirada en los trabajos del romanista Ernst Rabel, y otros instrumentos de soft law, como los Principios Europeos de Derecho de los Contratos, que intentan forjar una concepción más pragmática, realista e integral del contrato, superando el conceptualismo formalista de la codificación decimonónica. La aceptación de estos criterios no es, sin embargo, acrítica. Por ejemplo, Barros se resiste a pensar que todos los contratos sean asimilables a la compraventa, en los que no hay un deber de prestación que sea medible según estándares de cuidado y lo que se garantiza es un resultado. No ocurre lo propio, señala Barros con agudeza, con los contratos de prestación de servicios y, paradigmáticamente, en el mandato, donde no se puede evaluar el cumplimiento de las obligaciones del mandatario sin entrar en una discusión sobre los deberes de diligencia.

Decíamos al comenzar que este libro de homenaje, además de reflexivo, dialogante e innovador, es también multifacético. Como verán sus lectores, contiene una multiplicidad de estudios en diversas áreas y disciplinas jurídicas, y no sólo de derecho civil. Se ven artículos de filosofía y teoría del derecho, derecho constitucional y administrativo, y hasta de derecho procesal.

Podemos decir que Enrique Barros es también un jurista y un hombre polifacético. Quizás ello provenga de su primer interés por la filosofía, del que dan cuenta su tesis de licenciatura y luego su tesis doctoral, defendida con honores en la Universidad de München. Esta misma ductilidad para abordar problemas jurídicos de diversas áreas le ha permitido colaborar con el poder legislativo en muchas iniciativas legales, como la ley de filiación, la ley de matrimonio civil, la reforma al título XXXIII del libro I del Código Civil sobre corporaciones y fundaciones y la ley sobre competencia desleal.

Pero esta multiplicidad de intereses no se refiere sólo al estudio del derecho, sino que también se extiende a otras áreas, como el ejercicio de la abogacía y la labor gremial. Su gestión como presidente del Colegio de Abogados ha sido considerada por moros y cristianos como brillante. La aprobación de un nuevo Código de Ética Profesional, o de buenas prácticas como prefiere llamarlo Enrique, es un legado de extraordinaria importancia. Ya hemos mencionado su compromiso con la 
judicatura como abogado integrante de la Corte Suprema, a lo que hay que añadir su participación como árbitro de la Cámara de Comercio y de otras instituciones internacionales.

Más allá de lo jurídico, el profesor Barros ha colaborado con múltiples instituciones de bien público, entre ellas la Corporación Cultural de Santiago, que tiene a su cargo el Teatro Municipal, en cuya labor le habrá sido útil su pasión por la música selecta; la Bolsa de Comercio de Santiago y el Centro de Estudios Públicos, del que es consejero desde 1984 y, actualmente, su presidente. De allí que sea visto como una autoridad moral también en lo referido a la alta política.

Finalmente, nuestro libro es elegante pero a la vez cálido y acogedor. Así es también Enrique Barros, que tiene una elegancia y distinción francesa que seguramente le viene por línea materna. Pero esa elegancia no impone distancia ni genera frialdad en el trato; todo lo contrario: Enrique Barros es una persona afable, cálida, cordial, que se interesa por los demás, aunque los demás no siempre podamos aportarle mucho. Es un hombre que valora por sobre todo los afectos, el de sus alumnos, de sus colegas y amigos, pero en primer lugar de quienes conforman su familia: su señora, Rebeca, sus hijos y nietos.

Termino estas palabras expresando la gratitud que la academia y particularmente los civilistas le deben al profesor Enrique Barros Bourie. Es esa gratitud la que pretende expresar el libro que presentamos hoy.

Es cierto que a veces este tipo de obras puede sugerir que el homenajeado ya ha hecho todo lo que podía hacer y que ahora puede retirarse para gozar de un merecido descanso.

Sabemos que no será éste el caso de Enrique. Por el contrario, quisiéramos que la obra que presentamos se constituya en un estímulo para que siga poniendo sus extraordinarias dotes al servicio de la academia, el derecho y el bien del país. Como reza el viejo himno universitario, le decimos: Gaudeamus igitur, iuvenes dum sumus. Alegrémonos pues, Enrique, ¡aún somos jóvenes! 


\section{UN PROFUNDO AGRADECIMIENTO}

\section{Enrique Barros Bourie}

ada más dulce que recibir tanto afecto de quienes uno ha querido
en la vida. Los ensayos recogidos en este libro son un regalo entrañable.

He dedicado mucho tiempo a estudiar y enseñar. Pero quisiera decirles que no tienen nada que agradecer, porque mi vida académica ha sido mucho menos gratuita de lo que pudiera parecer.

Estudiar y escribir es una conversación con los pares académicos y con la comunidad jurídica. Enseñar no es un flujo en un solo sentido, sino una conversación ilustrada con alumnos que disciernen acerca de lo que es correcto y justo. No hay mejor control del exceso del "yo" que una sala de clases de esta escuela de derecho. Los alumnos, por intuición de espíritus jóvenes abiertos a entender y no a ser indoctrinados, distinguen al vuelo una opinión o doctrina indefendible.

Por eso, no es algo gratuito lo que agradecen; porque, en verdad, ¿qué habría sido mi vida si no hubiesen estado ustedes?

Infinitas gracias a Juan Carlos Marín y Adrián Schopf. Juan Carlos fue un destacado alumno de mi primer curso de derecho civil, en 1985; Adrián Schopf, compañero en la investigación jurisprudencial y en la edición de mi libro de responsabilidad. De ellos fue la iniciativa del libro que se presenta hoy y cuando supe de ella me sobrevinieron mucho pudor y una inmensa alegría. Su trabajo de editores y autores fue de una dedicación que me sobrecoge y que se muestra en la absoluta perfección de este libro.

Muchas gracias por este conjunto de magníficos ensayos escritos por muchos de los juristas que más admiro. Este libro habría sido un sueño hace algunas pocas décadas. Mi profundo agradecimiento por este presente. Es la mayor recompensa que puedo recibir como profesor de derecho.

Hace pocos meses, en momentos de sorpresiva amistad con la muerte, en completa soledad, sólo los afectos me vinieron a la concien- 
cia; por cierto, los referidos a lo más importante que me ha ocurrido, como es la mujer con que me casé y los cuatro hijos que me acompañan. Y a Norma, que por 35 años nos ha acompañado. A mi hijo Alberto, agradezco además su buenísimo estudio sobre derecho ambiental que me ha regalado.

Mi padre provenía del Chile profundo, de una familia agrícola empobrecida pero ilustrada. Mi madre francesa era reflexiva y protectora. Con mi hermano Gustavo entramos tarde al colegio. Él estudió ingeniería comercial, pero quedó arraigado a la tierra, y yo a la ciudad. Con mi padre aprendí a leer en El Mercurio y con mi madre, a sumar y restar.

Es importante tener razones para vivir. Los jesuitas fueron un gran modelo espiritual e intelectual desde la niñez. Nos introdujeron en la belleza de la fe y nos impulsaron a tratar de vivir por algo que valiera la pena.

Pertenezco a una generación que vivió el antiguo orden en el colegio y en los primeros años de universidad. Porque luego comenzamos a vivir los tiempos más dramáticos que haya vivido Chile.

Gracias, también, al puñado de profesores excepcionales que tuve en esta escuela. Gracias por la inclinación natural de mi espíritu, impulsada por mi padre, de quedarme en la universidad. Gracias a esta facultad, que por primera vez en su historia abrió en 1969 un concurso de ayudantes, que me permitió tener dedicación completa a la universidad a sólo cuatro meses de egresar.

Gracias a Jorge Millas por su luminosa y libre inteligencia y por la afectividad sin maneras, que me transmitió la fascinación por las humanidades y me impulsó a postular; a Antonio Bascuñán Valdés, porque amaba enseñar y contagiaba en mi época de ayudante su fascinación por la universidad.

En la Universidad de Chile aprendí que no importaba qué pensaba, sino ser riguroso y sincero en lo que se piensa. Sin encontrarme con muchas generaciones de alumnos despiertos habría sido más hipócrita, porque los jóvenes son el mejor antídoto contra la impostación y el narcisismo.

Gracias al profesor Arthur Kaufmann, una figura entonces señera en el derecho, que en tiempos menos cosmopolitas que los actuales recibió con afecto bíblico a un joven que con un alemán todavía balbuceante venía desde el fin del mundo a doctorarse en München. 
Gracias a Alemania, por la delicadeza con que nos recibió y por las muchas ventanas que nos abrió. Su propia tragedia era fundante de una forma virtuosa de convivencia. Fue uno de los períodos más fértiles y felices de nuestras vidas. Veníamos de un Chile violento y dividido por la ideología y la fuerza bruta. Luego de tantas desgracias, fue una bendición la oportunidad de sumergirse en 1974, y por cinco años, en un mundo académico tan libre y reflexivo.

Estuve un año encerrado sin levantar cabeza para lograr llenar vacíos de nuestra formación humanística. Valió la pena el esfuerzo de llegar a ser compañero de conversación con los ayudantes y compañeros de doctorado. Era un ambiente en que se vivía con radicalidad el desafío de pensar. Gracias especialmente a uno de ellos, brillante y generoso, Ulfrid Neumann, con quien hemos mantenido amistad por años.

Al regreso, gracias a los grupos de alumnos inquietos que me acompañaron en talleres de lectura voluntarios, que realizábamos en la torre de esta facultad, durante el exilio interno que viví en esta facultad al regreso de Alemania.

Agradezco haber encontrado entonces en el Centro de Estudios Públicos un lugar oxigenante donde desarrollar mi vocación pública desde la academia, lo que entonces no era posible desde esta facultad.

El CEP es una institución admirable que por más de 30 años ha mantenido un camino sin desvíos, gracias a un propósito genuinamente filantrópico de un grupo de empresarios, que han asegurado su independencia y un espíritu de servicio público de excelencia desde la sociedad civil. Es un honor haber sucedido en su presidencia a mis amigos Jorge Cauas y Eliodoro Matte. Con el correr de los años, se ha creado una cultura que conjuga la libertad de las humanidades y la seriedad en el estudio de políticas públicas razonables que contribuyan a un país más próspero y justo.

Fui a la profesión porque era la manera de ganarme la vida y asegurar mi libertad. Pero tuve también allí la fortuna de ser invitado por un gran abogado, don Carlos Torretti, un hombre de inteligencia afilada y de extrema dignidad personal.

Tuve la gracia de tener clientes que jamás ponen a su abogado en dilemas morales. El trabajo de abogado tiene la posibilidad de ser un arquitecto de relaciones de cooperación. También fui litigante, a mi pesar, porque nunca tuve gusto en el conflicto, ni el temple para tomar un juicio como un mero encargo profesional. 
El llamado como abogado integrante de la Corte Suprema permitió poner término a esa tarea y me permitió trabajar con una corte entonces excepcional, dedicada a decir el derecho y no a ser actora del proceso político, en una más de las bendiciones del clima político de la transición.

Agradezco también a la profesión buena parte de mis relaciones personales más profundas.

Nunca fui buen empresario de las leyes. Pero me alegra mucho que todos con quienes trabajé y se formaron conmigo hayan tenido una vida profesional plena y exitosa.

Estoy muy feliz de trabajar hoy con un pequeño grupo de jóvenes abogados y ayudantes que compartimos intereses y afectos, y que hacen muy feliz el momento en que llego a mi estudio. La vida ha sido muy generosa conmigo también en esta etapa de la profesión.

Aún recuerdo la fascinación con que enfrenté mi primer curso de derecho civil. Fue un ensayo de hacer más reflexivo el derecho civil. Lamento que la bota exegética haya sido tan dura en el primer examen. Con cada generación tuvimos una relación de respeto y calidez recíproca. Y cada vez que encuentro a mis ex alumnos veo en ellos las mil caras de la profesión.

El derecho es una construcción de la civilización. Mirado desde las humanidades, es un orden de nuestra vida en sociedad, que ha ocupado por dos mil años a miles de juristas, pero también a los más grandes filósofos, historiadores de las ideas y científicos sociales.

Cada generación de profesores de derecho deja su pequeña huella en esta larga historia. Antonio Bascuñán Rodríguez me envío hace un tiempo un impresionante listado de una centena de páginas con todos los profesores alemanes de derecho civil en los últimos siglos. Pocos, muy pocos, son los que aún recordamos.

Toda tarea humana es incremental, y en el derecho los cambios de paradigma se construyen a partir del aporte, anónimo con el tiempo, que hacen muchos estudiosos. Gracias a todos ellos por la sabiduría con que sin adivinarlo me han alimentado.

Gracias a Hernán Corral, José Miguel Huerta y a María Agnes Salah por sus palabras, a Daniela Accatino que estuvo impedida de llegar, a Adrián Schopf y Juan Carlos Marín, y a los juristas que han contribuido a esta obra excepcional. Su cariño me sobrecoge.

Gracias a todos ustedes por estar aquí con nosotros. EP 